\title{
CHARGED DUST IN THE EARTH'S MAGNETOSPHERE
}

\section{Physical and Dynamical Processes}

\author{
M. HORANYI \\ Supercomputer Computational Research Institute, Florida State University, Tallahassee, Florida, U.S.A. \\ H. L. F. HOUPIS \\ Space Physics Laboratory, Department of Atmospheric and Oceanic Science, The University of Michigan, \\ Ann Arbor, U.S.A. \\ and \\ D. A. MENDIS
}

Department of Electrical and Computer Engineering, University of California, San Diego, La Jolla, U.S.A.

(Received 12 November, 1987)

\begin{abstract}
We have considered the electrodynamic effects on small $\mathrm{Al}_{2} \mathrm{O}_{3}$ spherules dumped into the Earth's magnetosphere in large quantities during solid rocket propellant burns. The charges acquired by these grains in all regions of the terrestrial environment (plasmasphere, magnetosphere, and solar wind) are modest. Consequently electrodynamic effects are significant only at the lower end of the dust size spectrum $\left(R_{g} \lesssim 0.1 \mu \mathrm{m}\right)$. In that case, the electrodynamic forces conspire with solar radiation pressure to eliminate the grians from the magnetosphere in a comparatively short time. Although not studied here in detail, we anticipate a similar fate for fine micrometeoroids entering the Earth's magnetosphere, with the electrodynamic effects playing an even more important role.
\end{abstract}

\section{Introduction}

Small $(0.1-10 \mu \mathrm{m})$ sized $\mathrm{Al}_{2} \mathrm{O}_{3}$ spherules are dumped into the Earth's ionosphere/ magnetosphere during solid rocket motor burns used for transfer of satellites from low Earth to geosynchronous orbit. The flux from one such burn (No. of impacts $\mathrm{m}^{-2} \mathrm{yr}^{-1}$ ) could exceed the natural micrometeroid flux in that size (Mueller and Kessler, 1985). These authors also discussed the dynamic and orbital evolution of such particles in considerable detail taking into account the effects of gravity, gas drag, and solar radiation pressure. The one aspect that was not considered by them is the role of electromagnetic (Lorentz) forces that are experienced by these grains (which are necessarily electrically charged in the ambient plasma and radiative environment) as they move relative to the terrestrial magnetic field.

In this paper we will study the physical and dynamical processes associated with this electrostatic charging process. The time evolution of the grain orbits, and spatial distribution, as well as their magnetospheric residence times will be considered in detail in a subsequent paper.

\footnotetext{
* Paper dedicated to Professor Hannes Alfvén on the occasion of his 80th birthday, 30 May 1988.
} 


\section{Dynamies}

In the Earth-centered inertial frame, the motion of a charged dust grain of mass $m$, is governed by the equation

$$
m \ddot{\mathbf{R}}=\mathbf{F}_{\mathbf{G}}+\mathbf{F}_{\mathrm{LP}}+\mathbf{F}_{\mathbf{L}},
$$

where the three terms included on the right-hand side of Equation (1) are the gravitational, light-pressure, and Lorentz forces. In the region of interest, around the geosynchronous orbit, we may neglect the neutral gas and plasma (Coulomb) drags on the dust (Peale, 1966). Furthermore, we regard the interparticle distances to be larger than the Debye shieldinglength $\lambda$, so that interparticle Coulombinteractions are negligible.

To estimate the relative importance of these forces, we calculate their numerical values for a spherical grian with bulk density $\rho=1 \mathrm{~g} \mathrm{~A}^{-3}$ and radius $r_{g}=1$ (and 0.1$) \mu \mathrm{m}$, in the equatorial plane at geosynchronous altitude with zero velocity having an electrostatic potential $\phi$ of $10 \mathrm{~V}$ with respect to the ambient plasma. In this case, the gravitational force $F_{G}=9.4 \times\left(10^{-11}-10^{-14}\right)$ dynes.

The light pressure force is given by $F_{L P}=\pi r_{g}^{2} J_{0} Q_{p r} / c$, where $J_{0}$ is the solar energy flux at $1 \mathrm{AU}\left(\approx 1.36 \times 10^{6} \mathrm{ergan}^{-2} \mathrm{~s}^{-1}\right), Q_{p r}$ is the light scattering efficiency (assumed to be unity for both particles sizes) and $c$ is the speed of light. For the two grains in question $F_{L P}=1.42 \times\left(10^{12}-10^{-14}\right)$ dynes.

The Lorentz force is given by $\mathbf{F}_{L}=Q(\mathbf{E}+\dot{\mathbf{R}} \times \mathbf{B} / c)$, where $Q$ is the charge on the grain. For a small $\left(r_{g} \ll \lambda\right)$ 'isolated' $(\lambda \ll d$, where $d$ is the intergrain distance) grain $Q=\phi r_{g}$. Since we assume $\dot{\mathbf{R}}=\mathbf{0}$, we only need to consider the effect of $\mathbf{E}$, which has two components; $\mathbf{E}_{\text {co-rotation }}$ and $\mathbf{E}_{\text {cross-tail }}$. If we assume rigid co-rotation, $\mathbf{E}_{\mathrm{co}-}$ rotation $=-c^{-1}(\mathbf{\Omega} \times \mathbf{R}) \times \mathbf{B}_{0}\left(R_{E} / R\right)^{3}$ where $\Omega\left(=7.272 \times 10^{-5} \mathrm{rad} \mathrm{s}^{-1}\right)$ is the angular velocity of the Earth, $B_{0}(=0.31 \Gamma)$ is the equatorial surface magnetic field, and we have assumed the magnetic field to be a simple dipole field aligned with the rotatin axis. With the above values, $\mathbf{E}_{\text {co-rotation }} \approx 3.36 \times 10^{-6} \mathrm{~V} \mathrm{~cm}^{-1}$ at the geosynchronous orbit, and is pointed towards the Earth; since $\mathbf{\Omega}$ and $\mathbf{M}$ (the magnetic moment of the Earth) are antiparallel. The cross-field $\approx 2.5 \times 10^{-6} \mathrm{~V} \mathrm{~cm}^{-1}$, pointing in the dawn to dust direction. The maximum electric field clearly occurs at the dawn meridian, where $\mathrm{F}_{L} \approx 6.3 \times\left(10^{-14}-10^{-15}\right)$ dynes.

Comparing these numerical values, we conclude that for $1 \mu \mathrm{m}$ grains, the Lorentz force is a minor perturbation, but for grains with radii $\approx 0.1 \mu \mathrm{m}$ or smaller, the Lorentz force becomes comparable with the other two forces. It will thus play an important role in shaping the trajectories of these very small grains.

In order to follow the trajectory of a grain, one integrates Equation (1) with the current balance equation

$$
\dot{Q}=\Sigma I_{i}
$$

where $I_{i}$ are the various charging currents which will be discussed in the next section. Here it must be noted that due to the finite capacitance of the grain, the charge $Q$ at 
any point depends not only on the local plasma parameters and radiation field but also on its previous charging history.

The optical and physical parameters of these $\mathrm{Al}_{2} \mathrm{O}_{3}$ spherules are not too well known. However, we will consider two essentially extreme cases. In one case (type 1), we will assume the grain to have the scattering efficiency $Q_{p r}$ of conducting magnetite with $\rho \approx 3.2 \mathrm{~g} \mathrm{~cm}^{-3}$, whereas in the other case (type 2) we will assume it to have the scattering efficiency of dielectric olivine with $\rho \approx 2.2 \mathrm{~g} \mathrm{~cm}^{-3}$. Other material constants of these two types of grains (such as the photoelectron and secondary emission yields) will also be substantially different. These will be discussed in the next section.

\section{Charging Currents}

The right-hand side of Equation (2) contains all the charging currents to and from the surface of the grain. In the present study, we have the electron and ion (proton) thermal currents, secondary electron currents by electron and proton impact, backscattered and photoelectron currents. In the environment we consider, thermoionic and field emission currents can be shown to be negligible.

Below we summarize the expressions for the charging currents in the orbital motion limited approximation (Lafromboise and Parker, 1973). The thermal electron (e) and ion $(i)$ collection currents are

$$
I_{e, i}=e A \int_{\max (0, \pm e \phi)}^{\infty}\left[1 \pm\left(\frac{-e \phi}{E}\right)\right]\left(\frac{\mathrm{d} f_{e, i}}{\mathrm{~d} E}\right) \mathrm{d} E
$$

where $A\left(4 \pi r_{g}^{2}\right)$ is the grain surface area; $e$, the electron charge; $f_{e, i}$, the differential energy flux to the surface; $E$, the energy; and the,-+ signs correspond to electrons and ions, respectively. If we assume a Maxwellian distribution, Equation (3) can be integrated to yield

$$
I_{e, i}=e A n_{0}\left(\frac{k T_{e, i}}{2 \pi m_{e, i}}\right)^{1 / 2} \begin{cases}\exp \left(-\Psi_{e, i}\right), & \Psi_{e, i}>0, \\ 1-\Psi_{e, i}, & \Psi_{e, i}<0\end{cases}
$$

where $\Psi_{e, i}=\mp e \phi / k T ; k$ being the Boltzmann factor and $T_{e, i}$ is the temperature parameter in the Maxwell distribution.

This assumption is valid only when the velocity of the grain is much smaller than the thermal velocity of both the electrons and the ions. Otherwise one has to consider a drifting Maxwellian in the frame of the grain, and a more complicated expression for $I_{e, i}$, containing also the grain speed is obtained (see Whipple, 1981; Mendis, 1981). In the present calculations, it is appropriate to use Equation (4) to calculate the electron and ion collection currents both within the plasmasphere and the magnetosphere. It is also appropriate to calculate the electron collection current in the solar wind, but the more general expression for $I_{i}$ (Mendis, 1981) is required for the ion collection current in the solar wind. Consequently, the so-called radial gyro-phase drift associated with 
the orbital velocity modulation of the ion collection current by a finite capacitance grain (Hill and Mendis, 1980; Northrop and Hill, 1983) occurs only when the grain is in the solar wind, and is found to be a rather small effect even in this case.

The currents due to secondary electron emission by electron or proton impact, as well as backscattered electrons, can be calculated by incorporating the relevant yield function into Equation (3) as

$$
I_{s}^{k}=e A \int_{\max (0, \pm e \phi)}^{\infty}\left[1 \pm\left(\frac{-e \phi}{E}\right)\right] \delta^{k}(E \pm(-e \phi))\left(\frac{\mathrm{d} f_{k}}{\mathrm{~d} E}\right) \mathrm{d} E
$$

where $\delta^{k}$ is the appropriate yield function associated with the process $(k)$. The argument of $\delta^{k}$ is shifted due to the fact that the incoming particle looses or gains energy in approaching the grain through the potential field of the charged grain.

Equation (5) needs a further correction, because the secondary electrons will have a certain energy distribution, and in the case of an attractive potential only particles above the potential threshold will escape from the surface. In this calculation, we assume that the energy distribution of the secondary electrons is also a Maxwellian with a characteristic temperature parameter $T^{k}$. In this case, the correction factor for the attractive potential is $\left(1-\Psi_{\mathrm{s}}^{\mathrm{k}}\right) \exp \left(\Psi_{\mathrm{s}}^{\mathrm{k}}\right)$, where $\Psi_{s}^{k}=-e \phi / k T_{s}^{k}$.

For secondary electrons by electron impact, we use (Sternglass, 1954) the equation

$$
\delta^{S E E}(E)=7.4\left(\frac{E}{E_{M}}\right) \delta_{M}^{S E E} \exp \left[-2\left(\frac{E}{E_{M}}\right)^{1 / 2}\right]
$$

where $\delta_{M}$ and $E_{M}$ are material-dependent parameters. For particles of type 1, we use $\delta_{M}^{S E E}=1.5$ and $E_{M}^{S E E}=250 \mathrm{eV}$, and for type 2 , we use $\delta_{m}^{S E E}=2.4$ and $E_{M}^{S E E}=400 \mathrm{eV}$ (Whipple, 1981). For both types we used $k T_{s}^{S E E}=3 \mathrm{eV}$ (Whipple, 1981; Katz et al., 1977).

For secondary electrons by proton impact we use,

$$
\delta^{S E P}(E)=2 \delta_{M}^{S E P} \frac{\left(\frac{E}{E_{M M}^{S E P}}\right)^{1 / 2}}{\left(\frac{1+E}{E_{M}^{S E P}}\right)},
$$

where $\delta_{M}, E_{M}$ are the relevant material-dependent parameters. Due to the lack of laboratory data for both types of grains, we used $\delta_{M}=4.3$ and $E_{M}=40 \mathrm{keV}$, and for the energy distribution of the secondary electrons produced, we used $k T_{s}^{S E P}=3 \mathrm{eV}$ (Whipple, 1981; Katz et al., 1977).

For the secondary electrons by electron and proton impact, the yield function $\delta^{k}$ depends on the angle of incidence $\theta$ as well. The angular dependence is approximately $\sec (\theta)$, which when averaged over an isotropic distribution is just $\left\langle\delta^{k}(\theta)\right\rangle_{\theta}=2 \delta^{k}$ 
$(\theta=0)$, where $\delta^{k}(\theta=0)$ is the yield for normal incidence discussed above (Draine and Salpeter, 1979). This assumption of isotropic impact is valid for electrons in the plasmasphere, magnetosphere, and the solar wind, and it is also valid for ions in the plasmasphere and magnetosphere, but not in the solar wind. In that case the coefficient $z$ multiplying $\delta^{k}$ has to be replaced by $\propto$, where $1<\propto<2$.

For the backscattered electrons, we use a single step-function approximation

$$
\delta^{B S}(E)= \begin{cases}0, & E<E_{\min } \\ 0.3, & E \geq E_{\min }\end{cases}
$$

which is in reasonable agreement with the analytic expression given by Katz et al. (1977). Taking $E_{\text {min }}=100 \mathrm{eV}$ for the energy distribution of the backscattered electrons, we use (cf. Prokopenko and Lafromboise, 1980) the equation

$$
k T_{s}^{B E}=\left(0.45+2 \times 10^{-3} z\right)\left(k T_{e}+e \phi_{s}\right),
$$

where $\left(k T_{e}+e \phi_{s}\right)$ is the average incident electron energy and $z$, the atomic number of the target.

For all the secondary emission processes considered, we have neglected the possible decrease of secondary yield when the penetration depth of the bombarding particle becomes comparable to the size of the target, Given the average energies of the electrons and protons in the magnetosphere, this decrease is indeed small.

Finally, the photoelectron current is given by

$$
I_{p h}= \begin{cases}\pi r_{g}^{2} e f_{1}, & \phi<0 ; \\ \pi r_{g} e f_{1} \exp \left(\frac{-e \phi}{k T_{s}^{P}}\right), & \phi>0 ;\end{cases}
$$

where $k T_{s}^{p}(\approx 2 \mathrm{eV})$ is the average energy of the assumed Maxwellian distribution of photoelectrons and $f_{1} \approx 2.5 \times 10^{10} \chi \mathrm{s}^{-1}$, with $\chi \approx 1$ for type 1 grains and $\chi=0.1$ for type 2 grains (Mendis, 1981).

\section{The Plasma Environment}

In order to evaluate the various currents, we need to know the parameters of the plasma in which the grains are immersed. To describe the plasma environment of the Earth, we have followed Hill and Whipple (1985) with the modification that we include the local time-dependence of the plasma pause. Inside the plasma pause we assume thermal equilibrium between the ions and the electrons, with their density given by the empirical formula

$$
n(L)=10^{(15-L) / 3.5} \mathrm{~cm}^{-3},
$$

where $L$ denotes the magnetic shell, whose equatorial radius is $L$ Earth radii. The temperature is likewise given by the empirical relation 


$$
T(L)=0.09293 L^{2.7073} \mathrm{eV},
$$

when the temperature of the plasma given by (12) exceeds $1 \mathrm{eV}$, the plasma is considered to have two components, a hot one with $T$ given by Equation (12) and $n=1 \mathrm{~cm}^{-3}$, and a cold one with $T=1 \mathrm{eV}$ and density $1 \mathrm{~cm}^{-3}$ less than that given by Equation (11).

The boundary of the plasmapause is given (cf. Lyons and Williams, 1984) by

$$
L_{p p}=7.6 R_{E}\left(\frac{\sqrt{1+\sin \psi}-1}{\sin \psi}\right),
$$

where $\Psi$ is the local hour angle. Equation (13) defines a raindrop-like shape with $L_{p p}=3.8$ at 12 and 24 hours, and $L_{p p}=3.1$ and 7.6 toward 6 and 18 hous local time, respectively. Between the plasmapause and the magnetopause, we used the analytic expression fitted for the measured moments of the energy distribution of electrons and ions (Garrett and DeForest, 1979). The plasma is ocnsidered to have a bi-Maxwellian distribution. The data set is from the ATS -5 and ATS -6 satellites at geosynchronous orbit. Towards 18 hours local time, the plasmapause extends beyond the geosynchronous radius, but it leaves no obvious signature on the above satellite data. Perhaps this is because the detectors had a higher energy threshold, and it is for this reason that we kept the local time-dependence of the plasma pause. The bi-Maxwellian energy distribution, which is different for electorns and ions, depends on the daily geomagnetic activity index $A_{p}$ and on local time (Garrett and DeForest, 1979). In this study we kept $A_{p}$ constant at its average value of 120 . The magnetospheric plasma density is of the order of $1 \mathrm{~cm}^{-3}$, with characteristic energies of about $2 \mathrm{keV}$ for electrons and about $5 \mathrm{keV}$ for protons. The magnetopause which delineates the magnetospheric plasma, is described by a paraboloidal shape whose apex points toward the Sun. The subsolar point of the magnetopause is at $10.5 R_{E}$ and it opens up to $14.5 R_{E}$ at the terminators.

Beyond the magnetopause, we assume solar wind conditions with $n=5 \mathrm{~cm}^{-3}$ and $T \approx 10 \mathrm{eV}$.

\section{The Grain Charge}

The current potential characteristics are plotted in Figures $1 \mathrm{a}-\mathrm{c}$ ), for the three different plasma regimes. Within the plasma sphere we have high plasma densities and relatively low thermal energies. For negative potentials, the proton collection current, the secondary electron current due to proton impact, and the photoelectron current are the major contributors to the total current. For positive potentials, the electron collection current dominates. The peak in the secondary electron current by electron impact is a result of the competition between two opposing tendencies. On the one hand, as the grain potential increases, more electrons with energies closer to the optimum for secondalry emission impact the surface. On the other hand, it becomes harder for the secondary electrons to leave the surface. 
Magnetosphere at $T=18 \mathrm{H}$

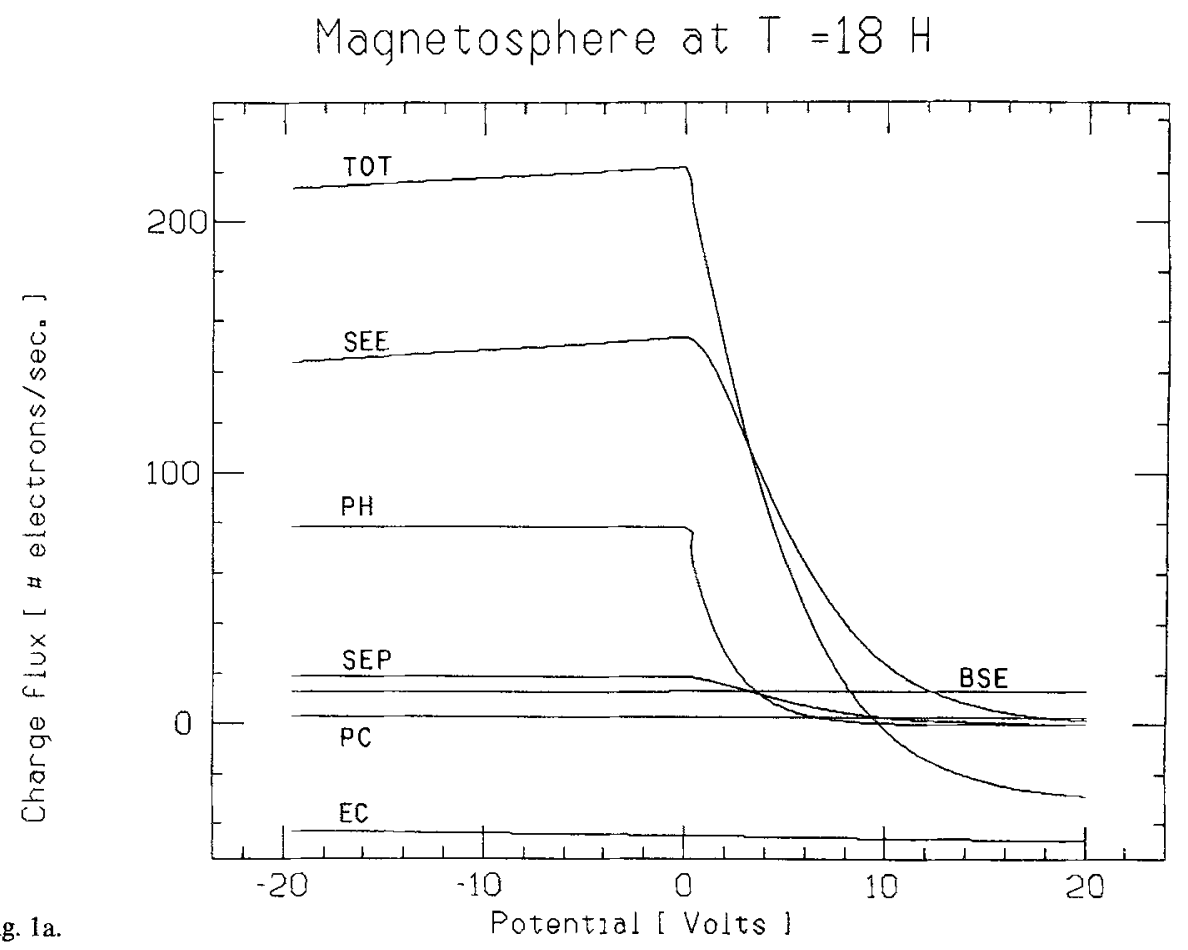

Fig. 1a.

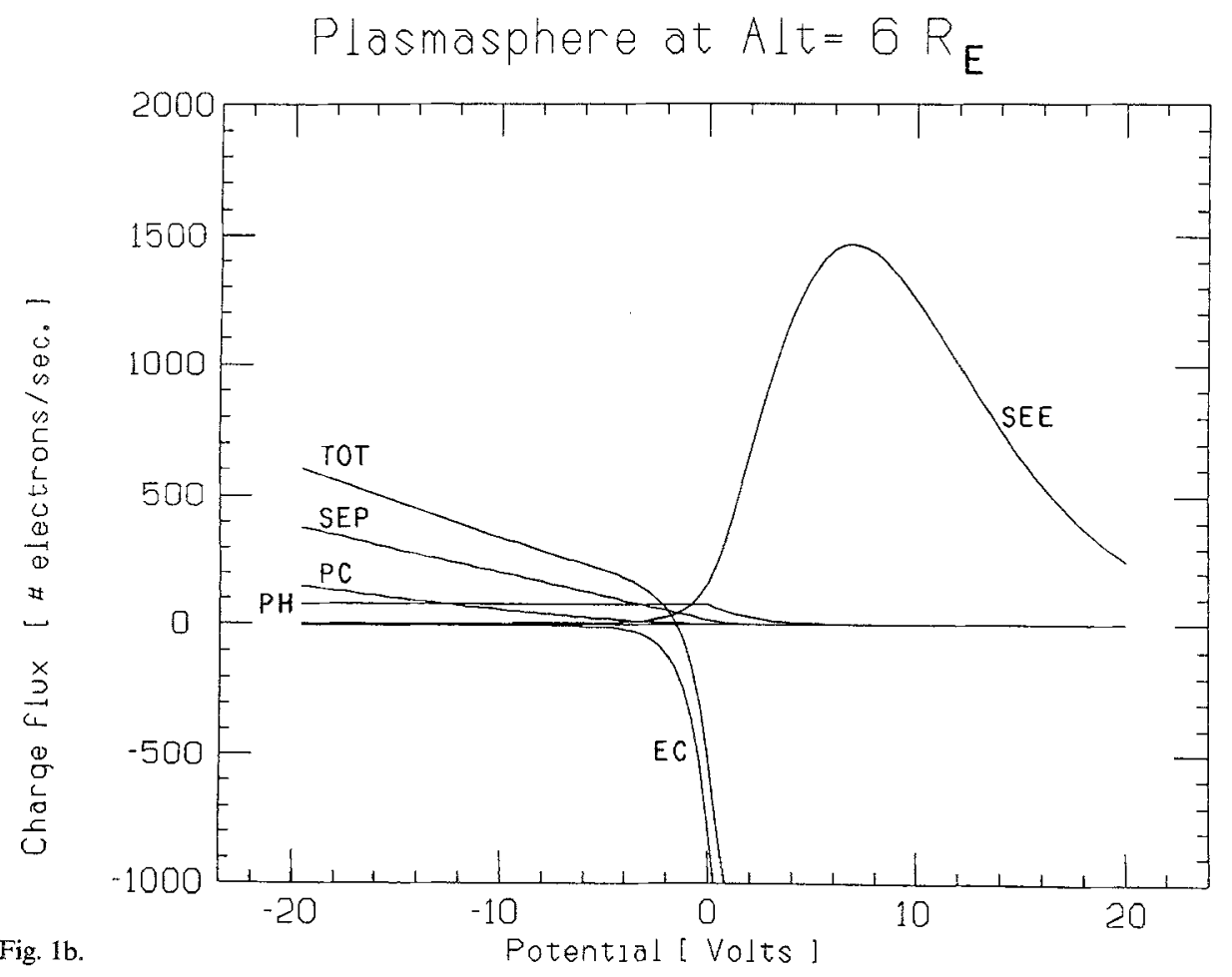




\section{Solar wind}

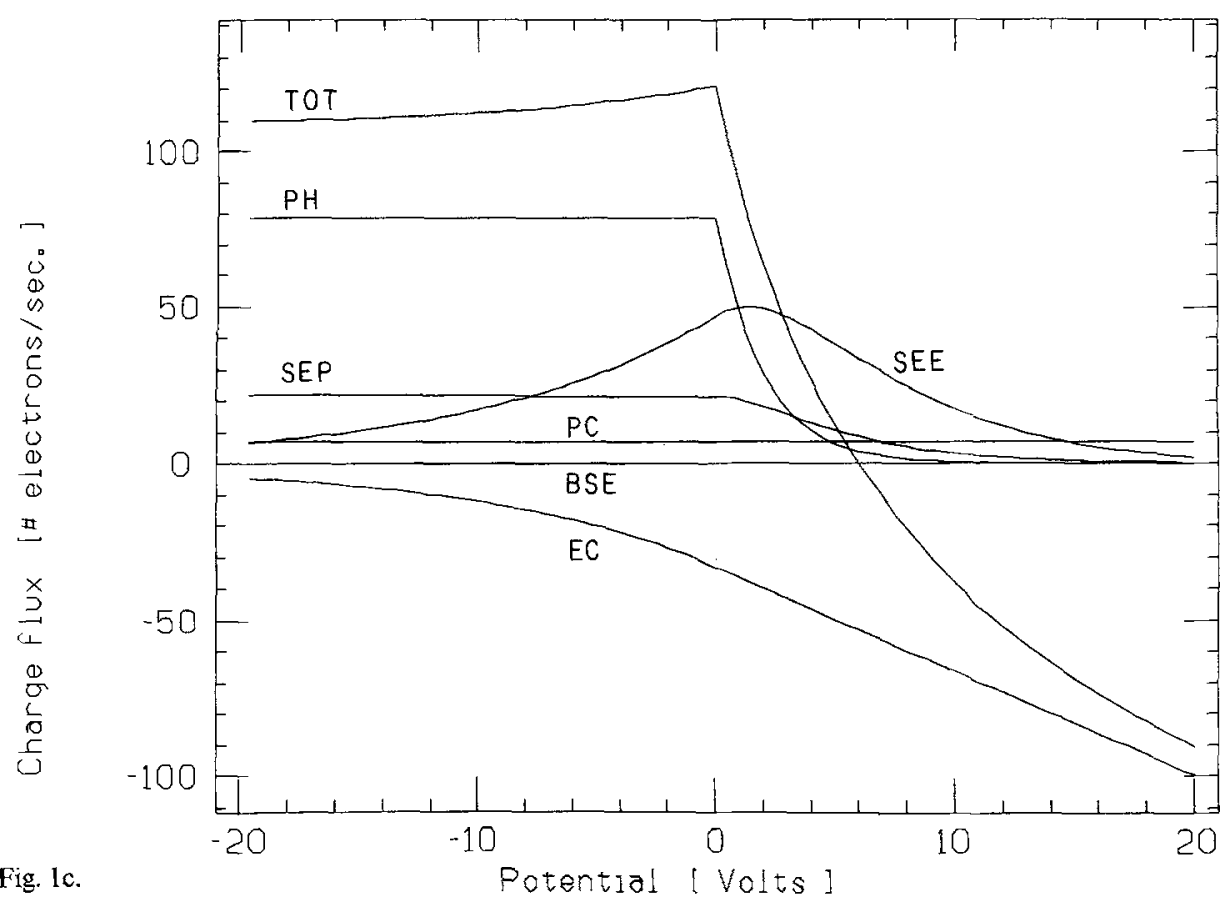

Fig. 1. The current-potential curves the three plasma regimes, for a grain of radius $1 \mu \mathrm{m}$. (a) Within the plasma sphere at a geocentric distance of $6 R_{E}$. (b) In between the plasmapause and the magnetopause, at $18 \mathrm{hr}$ local time. (c) In the solar wind. In each case, $E C, P C$, and $P H$ denotes the electron collection, the proton collection and photo-emission current, respectively, while $S E E, S E P$, and $B S E$ denotes the secondary electron currents due to electron impact, and ion impact, and the backscattered electron currents. Also the currents are measured in units electron charge per sec, with + sign indicating electrons leaving from and - sign indicating electrons arriving at the grain.

In the magnetosphere, outside the plasmapause, the polasma has low densities and high energies. The addition or subtraction of energy in moving through the grain potential is negligible in comparison with the characteristic particle energies. All currents are almost constant, but secondary electron currents due to electron and proton impact, as well as the photoelectron current rapidly decrease to zero at positive grain potentials due to the fact that the associated emitted electron distribution are soft.

In the solar wind the plasma has both low densities and relatively low temperatures. The major charging currents are due to electron collection, photoemission and secondary electron emission by electron impact.

If we keep a grain long enough in a given plasma and radiative environment, it will achieve an equilibrium potential (and associated charge), when the net current to it is zero. The time history towards this equilibrium potential is shown in Figure 2. Figures 
Type 1

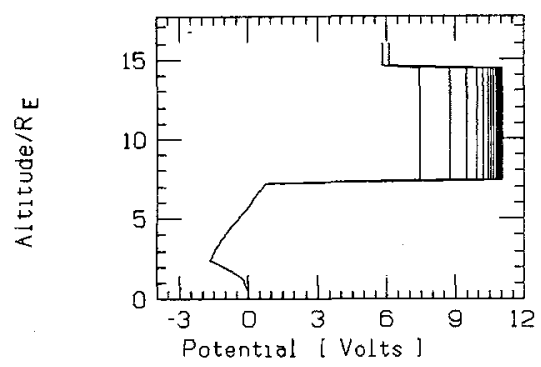

(a)

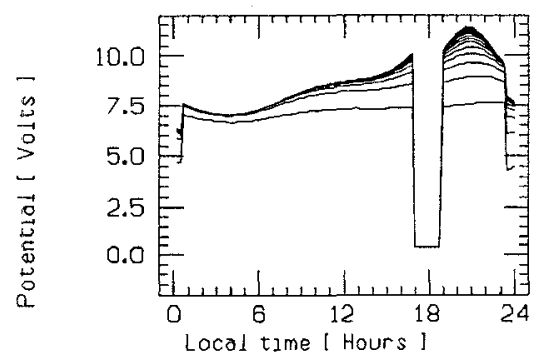

(c)
Type 2

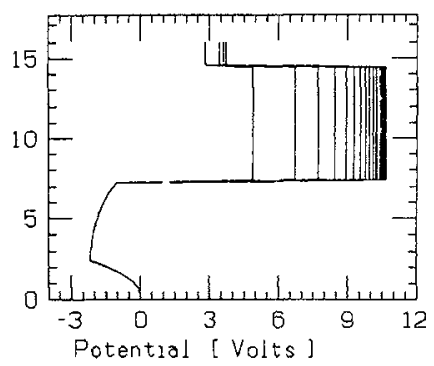

(b)

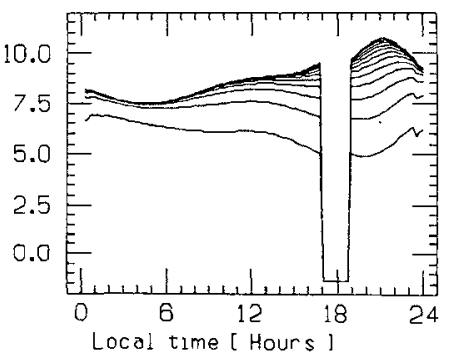

(d)

Fig. 2. The time history of the evolution of the potential of a grain of radius $0.1 \mu \mathrm{m}$ to its equilibrium value: panels (a) and (b) show the variation with altitude at $18 \mathrm{hr}$ local time for grains of type 1 (conductors) and for grains of type 2 (dielectrics), respectively, while panels (c) and (d) show the variation with local time, at geosynchronous orbit for type 1 and type 2 grains, respectively. The individual curves correspond to times 10 min apart.

2(a-b) show this for the two types of grains as a function of altitude, at 18 hours, local time. The individual curves correspond to times $10 \mathrm{~min}$ apart. The grain has a small negative potential at low altitudes, decreasing with altitude until the appearance of the second component of the plasma in the plasmasphere. It then begins to increase with increasing altitude, but still remains negative throughout the plasmasphere. Also, within the plasmasphere, due to the high plasma densities, the grain attains its equilibrium potential rapidly (within minutes).

At the altitude of $7.6 R_{E}$ we reach the magnetopause, where it takes almost two hours to reach the equilibrium potential for type 2 grains and a somewhat shorter time for type 1 grains. The solar wind is reached at an altitude of $14.5 R_{E}$ where it takes less than $20 \mathrm{~min}$ for type 1 and less than $\mathbf{4 0} \mathrm{min}$ for type 2 grains to achieve equilibrium potential.

On Figures $2(\mathrm{c}-\mathrm{d})$ are plotted the equilibrium potentials vs local time at geosynchronous orbit. For both type 1 and type 2 grains, the sudden decrease at around 18 hours local time is due to the fact that the geosynchronous orbit cuts into the plasmasphere around that direction. For type 1 grains the photoelectron current is about 10 times larger than that for type 2 grains which explains the jump in the equilibrium 
potential around midnight when the grains are in the Earth's shadow. (In the present study we used only the geometrical shadow of the Earth, while the optical depth of the atmosphere was neglected.)

It should also be mentioned that we have carefully checked for the possibility of multiple equilibrium potentials as suggested, for example by Meyer-Vemet (1982). When average geomagnetic conditions are used, as we have done, they do not exist. The possibility that they do exist at extreme geomagnetic conditions cannot be ruled out.

As the grains move along their trajectories, they may not spend enough time in a given plasma environment to achieve the local equilibrium potential. It is necessary to integrate Equation (1) simultaneously with Equation (2) to find the actual potential reached at any given point on the orbit. To illustrate this, we consider a grain moving with Kepler speed at the geosynchronous orbit, where the effects of light pressure and the Lorentz force has been turned off in order to have a periodic orbit. The light curve in Figure 3 shows the variation of equilibrium potential, discussed earlier, with local time. The dark curve shows the variation of the actual grain potential with local time once it has achieved a 'steady' periodic potential. It is seen that as the grain dips into the plasmasphere around 18 hours, it achieves its equilibrium potential almost instantly. This is due to the large plasma density there. However, as it emerges from the plasma

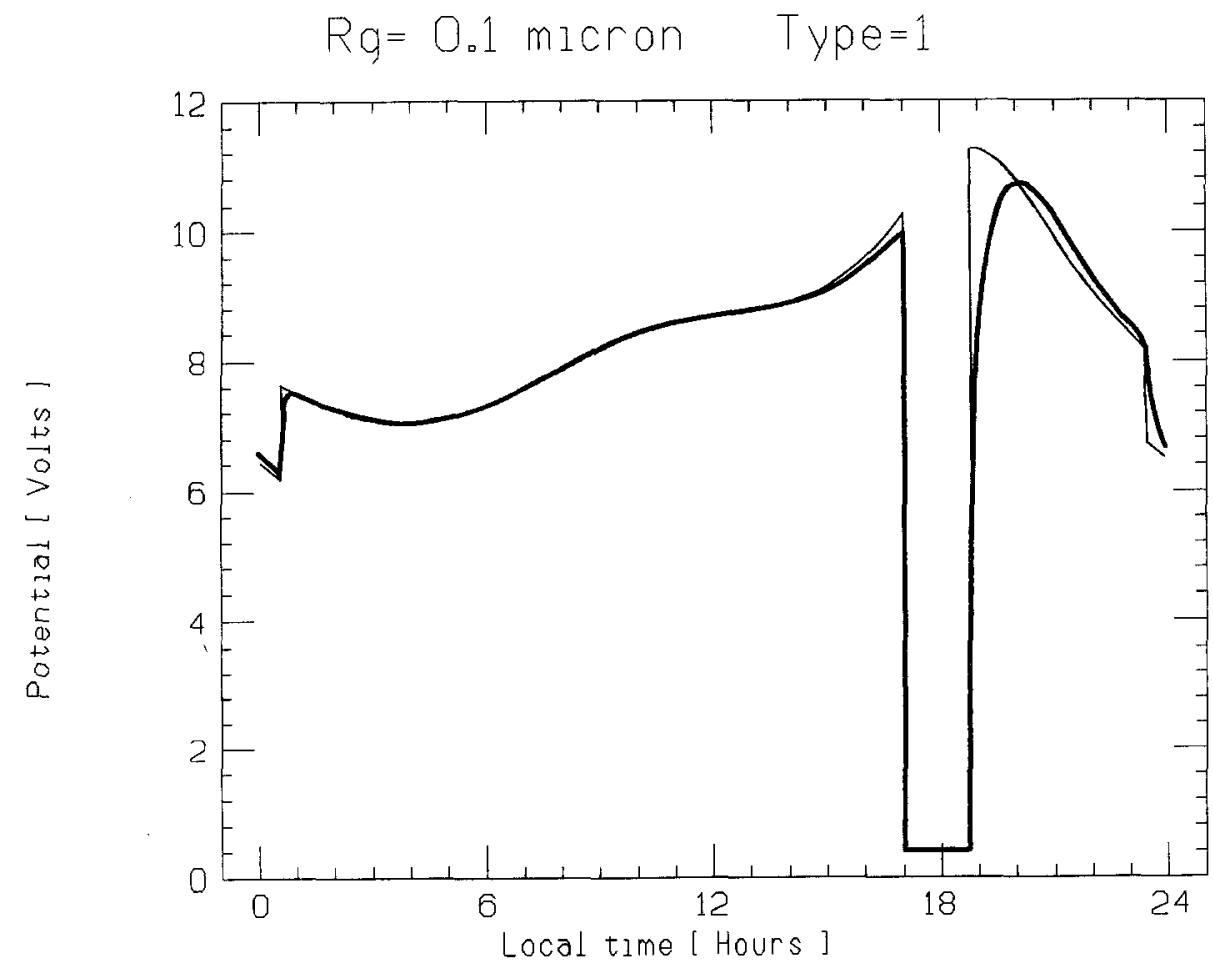

Fig. 3. Variation with local time of the equilibrium potential (light curve) and the actual potential achieved (dark curve) by a type 1 grain of radius $0.1 \mu \mathrm{m}$ moving in a circular orbit at geosynchronous orbit. 
sphere into the magnetosphere (where the plasma density is much smaller) it takes time to approach the local equilibrium potential. Indeed it does so only after it has also entered and re-emerged from the Earth's shadow around 24 hours.

\section{Orbital Evolution}

Figure 4(a) shows the orbit of a grain of type 2 (dielectric) of radius $0.1 \mu \mathrm{m}$ injected into the magnetosphere inside the geosynchronous orbit $\left(R=0.8 R_{g s}\right)$ at noon. The lighter curve corresponds to the case when the Lorentz force is neglected (i.e., $Q=0$ ), while the darker curve corresponds to the case when the Lorentz force is included $(Q \neq 0)$. The dominant effect of radiation pressure is clear in both cases. The large-amplitude oscillation of ghe grain's orbital eccentricity caused by solar radiation pressure for the case $Q=0$ is well known to be the dominant effect in the evolution of the orbit (Shapiro, 1963; Peale, 1966). This is also seen to be the main effect in the dynamics of the charged grains. The main difference is in the orientation of the orbits. In the case when $Q=0$, the peregee and apogee are along the E-W meridian. The evolution continues until a maximum eccentricity is reached after which the process is reversed and repeated, unless the grain hits the Earth's atmosphere and is lost. In the case when $Q \neq 0$, the apogee and peregee are not along this E-W meridian but displaced from it by about $18^{\circ}$. Also, both grains $(Q=0$ and $Q \neq 0$ ) hit the Earth in about the same time period; $(T(Q=0)=6.01$ days, $T(Q \neq 0)=5.91$ days $)$, although they do so at different points. The uncharged grain hits the Earth at the evening (18 hr) meridian, whereas the charged hits the Earth around the noon (12 hr) meridian.

Figure 4(b) shows the orbit of a grain of type 2 of radius $0.1 \mu \mathrm{m}$, which is injected into the magnetosphere outside the geosynchronous orbit $\left(R=1.2 R_{g s}\right)$ at noon. As before, the lighter curve corresponds to the case $(Q=0)$ while the darker one corresponds to the case $(Q \neq 0)$. The situation is qualitatively similar to the previous case, but due to the larger Lorentz forces experienced by the charged grain, its orbit differs more from the orbit of the charged grain than in the previous case. Note that in this case the apogee and peregee lie on a line displaced from the E-W meridian by about $25^{\circ}$. Also in this case, while the time taken by the uncharged grain to hit the Earth is about 6.08 days (which is in fact slightly larger than that for the unchanged grain launched from $R=0.8 R_{g s}$ ), the time taken by the charged grain is significantly smaller ( 4.54 days). Clearly, while the residence times of the grains that are charged are smaller than their uncharged counterparts, the discrepancy in the residence times of charged and uncharged grains (of equal physical and optical properties) launched from the same point will increase as its geocentric distance increases.

Finally in Figure 5 are shown the orbits of type 1 (conducting) grains of radius $0.1 \mu \mathrm{m}$ injected into the magnetosphere at noon from $R=0.8 R_{g s}$ and from $R=1.2 R_{g s}$. As before, the lighter curves correspond to the uncharged grains, while the darker curves correspond to the charged grains. The radiation pressure effect on these grains are much larger than in the case of the type 1 (dielectric) grains. Consequently, the orbital evolution due to this effect is much larger, and the difference between the orbits of the 

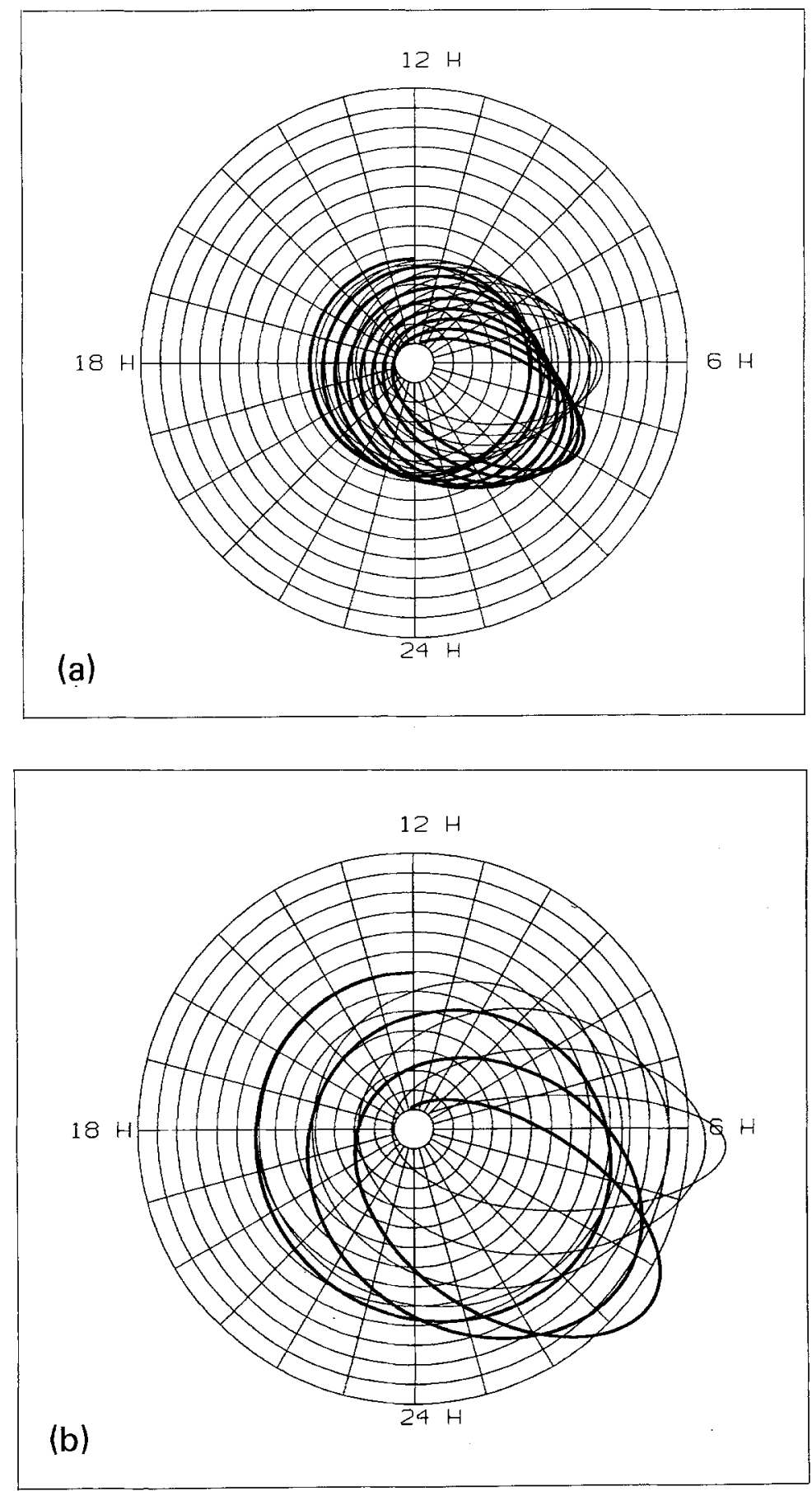

Fig. 4. Orbits of type 2 grains or radius $0.1 \mu \mathrm{m}$ injected into the magnetosphere at $12 \mathrm{hr}$ local time, (a) inside the synchronous orbit (at $R=0.8 R_{s}$ ) and (b) outside the synchronous orbit (at $R=1.2 R_{s}$ ). In each case, the light curve denotes an uncharged grain $(Q=0)$, while the dark curve denotes a charge grain. 


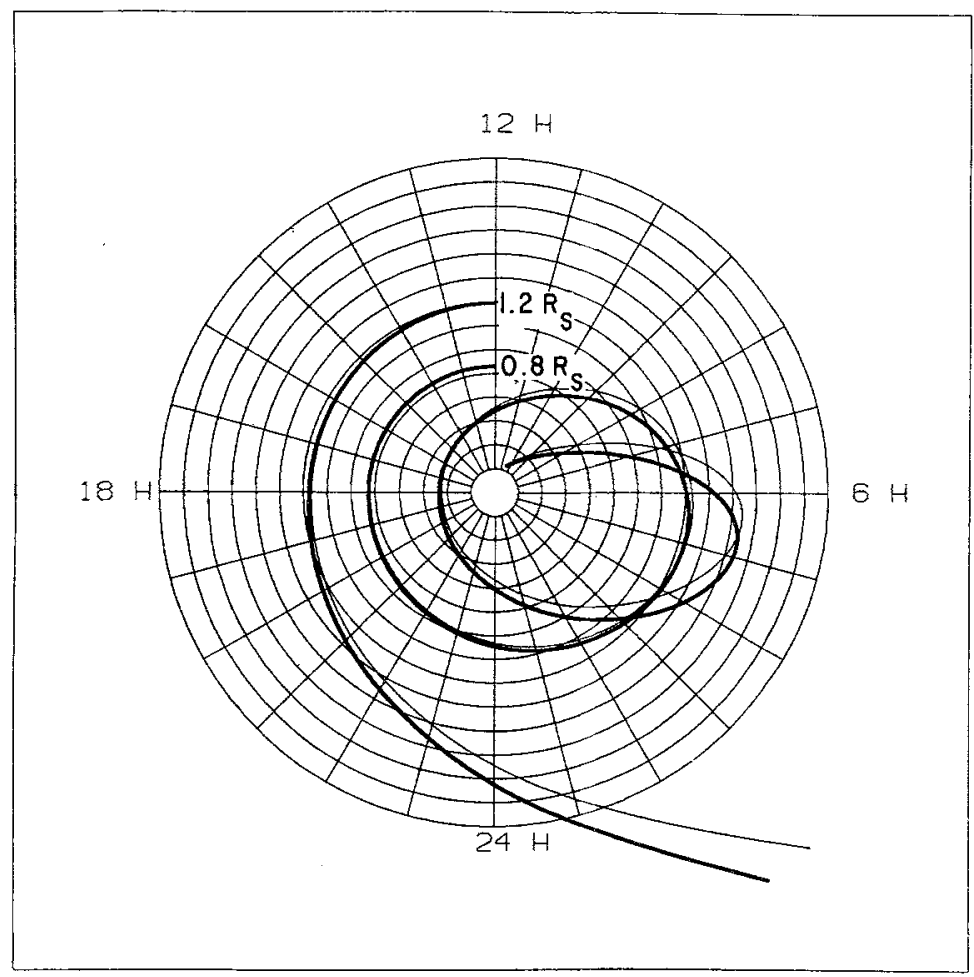

Fig. 5. Same as Figures $4(\mathrm{a}-\mathrm{b})$, but foa a grain of type 1 with radius $0.1 \mu \mathrm{m}$. The orbits corresponding to injection both at $0.8 R_{s}$ and at $1.2 R_{s}$ are shown in the figure.

charged and uncharged grains is smaller than in the case of the dielectric grains. Thus, the discrepancy of the residence times between the charged and uncharged grains is also small. Both grains injected at $R=0.8 R_{g s}$ crash to Earth at about the same point, after about the same residence time $(T(Q=0)=1.88$ days, $(T(Q \neq 0)=1.86$ days $)$. Both grains injected at $R=1.2 R_{g s}$ leave the magnetosphere (at $R=20 E_{E}$ ) in the very first orbit $(T(Q=0)=1.12$ days, $(T(Q \neq 0)=1.14$ days $)$.

\section{Discussion}

The central aim of our ongoing study is to assess the role of electromagnetic (Lorentz) forces that are experienced by fine dust particles that are injected into the Earth's magnetosphere by natural (micro-meteoroid flux) and by artificial (solid rocket propellant burns) processes. In the present paper we have confined our attention to the latter case. In order to make some progress in this initial study, we have used a rather simple model of the particles and fields environment of the Earth, and confined our attention to grain orbits in the equatorial plane. We have considered all the important charging currents on to the grains and shown that the electric charge acquired by the grains is 
rather modest. It varies typically from a few volts (negative) within the plasma pause to about $+10 \mathrm{~V}$ in the region between the plasmapause and the magnetopause, to a few volts (positive) in the solar wind. Under these circumstances, the solar radiation pressure-driven oscillation of the orbital eccentricity is still the dominant dynamical feature of the orbital dynamics. However, for the smallest grains $\left(r_{g} \approx 0.1 \mu \mathrm{m}\right)$, the electromagnetic (Lorentz) forces associated with the grain motion through the magnetized plasma, significantly changes the nature of the orbits, particularly if the grains are dielectric. The magnetospheric residence times of the grains are also significantly decreased by the charging, the effect being more pronounced for grains injected at larger geocentric distances.

While we recognize that the relevant plasma distributions are highly dependent on the geomagnetic index $A_{p}$ we have kept $A_{p}$ constant at its average value of 120 in this calculation. Since increased $A_{p}$ during disturbed solar wind conditions leads to higher magnetospheric temperatures (Garrett and DeForest, 1979), we expect that the electrodynamic effects on the grains during such times would be larger, leading to even shorter residence times than those corresponding to $A_{p}=120$. In a subsequent paper we propose to investigate that effect.

Besides the artificial injection of grains in the vicinity of the synchronous orbit discussed above, there is also the natural injection of micrometeoroids into the magnetosphere. Since these are injected with a relative velocity equal to about the escape velocity and are already charged as they penetrate the magnetopause, we expect that the electrodynamic effects they experience would be quite large (perhaps even comparable to the effects of radiation pressure). Consequently, they may be lost (either by impact with the Earth) or by ejection from the magnetosphere, even faster. We propose to study the question too in the subsequent paper.

There we will study the evolving distribution functions as well as the differential residence times (with grain radius and location of injection) for both the artificial and natural populations. We will also consider the steady-state distributions assuming continuous injection at the appropriate rates.

Another complication that has to be taken into account is the finite inclination of the spin axis of the Earth to the magnetic axis, which necessitates the consideration of 3-D distributions. In this case grains injected in the equatorial plane will be quickly dispersed normal to it by the electrodynamic forces they experience in that direction.

While we prognose a detailed quantitative study of all these in the subsequent paper, it is already apparent qualitatively from the present study that the electrodynamic effects on charged grains in the Earth's magnetosphere appears to conspire with the solar radiation pressure effects to eliminate fine dust from it.

\section{Acknowledgements}

One of us (M.H.) acknowledges support from DOE under agreement No. DE-FCOS-85ER25000. The simulations were done on the Cyber 205 at the Super- 
computer Computational Research Institute, Florida State University, which donated part of the computation time.

\section{References}

Draine, B. T. and Salpeter, E. E.: 1979, Astrophys. J. 231, 77.

Garrett, H. B. and DeForest, S. E.: 1979, J. Geophys. Res. 84, 2083.

Hill, J. R. and Mendis, D. A.: 1980, The Moon and Planets 23, 53.

Hill, J. R. and Northrop, T. G.: 1983, J. Geophys. Res. 88, 1.

Hill, J. R. and Whipple, E. C.: 1985, J. Spacecraft Rockets 22, 245.

Katz, I., Parkes, D. E., Mandell, M. J., Harvey, J. M., Brownell, D. H., Wang, S. S., and Rotenberg, M.: 1977, NASA CR-135256.

Lafromboise, J. G. and Parker, L. W.: 1973, Phys. Fluids 16, 629.

Lyons, L. R. and Wiliams, D. J.: 1984, Quantitative Aspects of Magnetospheric Physics, D. Reidel Publ. Co., Dordrecht, Holland, p. 79.

Mendis, D. A.: 1981, in F. D. Kahn (ed.), Investigating the Universe, D. Reidel Publ. Co., Dordrecht, Holland, p. 353.

Meyer-Vermet, N.: 1982, Astron. Astrophys. 105, 98.

Mueller, A. C. and Kessler, D. J.: 1985, Adv. Space Res. 5 (No. 2).

Northrop, T. G. and Hill, J. R.: 1983, J. Geophys. Res. 88, 1.

Peale, S. J.: 1966, J. Geophys. Res. 71, 911.

Prokopenko, S. M. L. and Laframboise, J. G.: 1980, J. Geophys. Res. 85, 4125.

Shapiro, I. I.: 1963, in M. Roy (ed.), Dynamics of Satellites, Springer-Verlag, Berlin, p. 257.

Sternglass, E. J.: 1954, Sci. Pap. 1772, Westinghouse Research Laboratory, Pittsburg, PA.

Whipple, E. C.: 1981, Rep. Progress Phys. 44, 1197. 